\title{
Review: in people with schizophrenia, lithium is ineffective as sole therapy, while evidence on augmenting antipsychotics with lithium is inconclusive
}

Leucht S, Kissling W, McGrath J. Lithium for schizophrenia revisited: a systematic review and meta-analysis of randomized controlled trials. J Clin Psychiatry 2004;65:177-86.

\section{$Q$ Is lithium, as a sole agent or an adjunct to antipsychotic drugs, effective in the treatment of schizophrenia?}

METHODS

L

Design: Systematic review with meta-analysis

Data sources: Cochrane Schizophrenia Group Register of RCT (searched to March 2002). Authors of included studies were contacted to obtain original patient data.

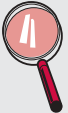

Study selection and analysis: Eligible studies were RCTs, in any language, examining lithium as a sole or supplementary treatment in people with schizophrenia or related disorders. Study quality was assessed: only studies with a low or moderate risk of bias (according to categories described in the Cochrane Collaboration Handbook) were included. For dichotomous data, the relative risk (RR) was calculated for each study; for single studies the Mantel-Haenszel fixed effect model for homogenous outcomes and the Der-Simonian-Laird random effects model for heterogeneous outcomes were used; for the analysis of continuous outcomes tentative standardised mean differences were calculated. Heterogeneity was examined by inspecting graphs and a $\chi_{2}$ test of heterogeneity. People with schizoaffective disorder or predominant affective disorder were excluded in a second analysis to rule out any superiority of lithium due to the improvement of manic or depressive symptoms.

Outcomes: Number of individuals without a clinically significan improvement: as defined in the original study, or $<50 \%$ reduction of the score of a rating scale-for example, the Brief Psychiatric Rating Scale (BPRS). Small (<20\% BPRS score reduction) and intermediate $(<35 \%$ BPRS score reduction) reductions were also analysed. Number of participants leaving studies early: as defined by the original studies.

\section{MAIN RESULTS}

Twenty RCTs met inclusion criteria $(n=611)$. Lithium alone versus placebo: there was no significant difference in clinical improvement between lithium and placebo $(n=54, \mathrm{RR} 1.1,95 \%$ CI 0.7 to 1.8 ). There was no significant difference in people leaving the study early between lithium and placebo $(n=65$, RR $1.1,95 \%$ CI 0.3 to 4.4). Lithium alone versus antipsychotics: there was no significant difference in number of people without a clinically significant response between lithium and antipsychotics $(n=80, R R$ $1.395 \%$ CI 0.8 to 2.2 ). However, significantly more people taking lithium left the study early $(\mathrm{n}=270, \mathrm{RR}=1.8,95 \%$ CI 1.1 to 2.9$)$. Four trials found this difference to be due to treatment failure of lithium ( $\mathrm{n}=178, \mathrm{RR} 3.0,95 \%$ CI 1.2 to 7.8$)$. Lithium versus placebo as an adjunct to antipsychotics: lithium supplementation significantly reduced the risk of "no clinical response" compared with placebo $(\mathrm{n}=244, \mathrm{RR}=0.8 ; 95 \% \mathrm{CI}=0.7$ to 0.9$)$, however this difference was not consistent across response thresholds. When people with prominent affective symptoms were excluded from the analysis the advantage was not significant $(p=0.07)$. In addition,

For correspondence: Stefan Leucht, Department of Psychiatry and Psychotherapy, Technische Universitat Munchen, Klinikum rechts der Isar, Ismaningerstr, Muenchen, Germany; Stefan.Leucht@lrz.tum.de

Sources of funding: the German Federal Ministry for Education and Research. significantly more participants left studies early when taking lithium $(\mathrm{n}=320, \mathrm{RR}=2.0 ; 95 \%$ CI 1.3 to 3.1$)$.

\section{CONCLUSIONS}

Treatment of schizophrenia with lithium alone is not effective. Results of lithium augmentation are inconclusive. Any benefit may be outweighed by a lower acceptability of treatment.

\section{Commentary}

n everyday clinical practice, lithium is used in patients with schizophrenia for augmentation of neuroleptic efficacy on psychotic (core) symptoms, treatment of aggression and excitement, and treatment of affective symptoms. ${ }^{1}$ Reviews of the literature state that affective symptoms need not be present for patients with schizophrenia to respond to lithium. ${ }^{2}$

Current schizophrenia treatment guidelines codify rather different indications for lithium augmentation: adjunctive use in patients displaying aggressive behaviour (without sufficient empirical evidence); ${ }^{3}$ augmentation of clozapine as second line strategy in patients with continuing prominent positive symptoms, and adjunctive use for any neuroleptic medication in patients displaying agitation or aggression; ${ }^{4}$ or combination with antipsychotics for persisting positive symptoms, excitement, aggression, or affective symptoms. ${ }^{5}$

Most studies on lithium in schizophrenia are case reports; randomised double blind trials are few. To our knowledge, Leucht et al present the first meta-analysis. Not surprisingly, lithium was shown to be ineffective as a sole agent. However, the efficacy of lithium augmentation of antipsychotic medication proved to be less than expected and may be mostly related to affective symptoms. This is an important finding, as clinical practice and also practice guidelines seem to mirror a rather biased view of the published studies and case reports.

The clinical implications of this meta-analysis are as follows: on the one hand there appears to be no clear empirical evidence that lithium augmentation has a positive effect in schizophrenia except for affective symptoms, on the other hand there seems to be no convincing alternative strategy, as the combination with other agents such as valproate or benzodiazepines also lacks sufficient empirical evidence.

Given the inconclusive results of previous studies, it would be highly desirable if-as Leucht et al suggest-a larger, double blind, randomised trial on lithium augmentation was conducted. Depending on its results, we might be prompted to reconsider our clinical practice and to adapt the guidelines, and maybe this would lead to a more frequent use of ECT instead.

Stefan Bender, MD, Alexandra Dittmann-Balcar, PhD Westfälische Klinik Marsberg, Marsberg, Germany

1 Bender S, Linka T, Wolstein J, et al. Safety and efficacy of combined clozapine-lithium pharmacotherapy. Int J Neuropsychopharmacology 2004; 7:59-63

2 Christison GW, Kirch DG, Wyatt RJ. When symptoms persist: choosing among alternative somatic treatments for schizophrenia. Schiz Bull $1991 ; 17: 217-45$

3 Lehman AF, Lieberman JA, Dixon LB, et al; American Psychiatric Association. Steering Committee on Practice Guidelines. Practice guideline for the treatment of patients with schizophrenia (2nd edition). Am J Psychiatry 2004;161(Suppl 2):1-56.

4 McEvoy JP, Scheifler PL, Frances A, eds. The Expert Consensus Guideline Series: Treatment of Schizophrenia 1999. J Clin Psychiatry 1999;60(Suppl 11).

5 Deutsche Gesellschaft für Psychiatrie, Psychotherapie und Nervenheilkunde (ed). Praxisleitlinien in Psychiatrie und Psychotherapie; Band 1: Behandlungsleitlinie Schizophrenie. Darmstadt: Steinkopff, 1998. 